\title{
Comparison of the Effects of Telbivudine and Entecavir Treatment on Estimated Glomerular Filtration Rate in Patients with Chronic Hepatitis B
}

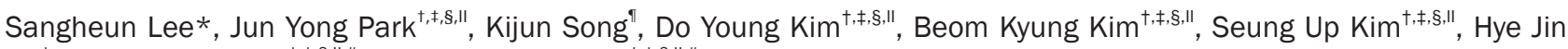

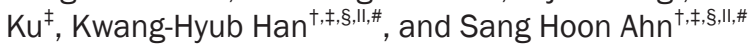 \\ ${ }^{*}$ Department of Internal Medicine, International St. Mary's Hospital, Catholic Kwandong University College of Medicine, Incheon, ${ }^{\dagger}$ Department \\ of Internal Medicine, ${ }^{\ddagger}$ Institute of Gastroenterology, ${ }^{S}$ Liver Cancer Special Clinic, Yonsei University College of Medicine, "Liver Cirrhosis \\ Clinical Research Center, "Department of Biostatistics, Yonsei University College of Medicine, and \# Brain Korea 21 Project for Medical Science, \\ Seoul, Korea
}

Background/Aims: The aim of this study was to evaluate the estimated glomerular filtration rate (eGFR) during telbivudine (LdT) versus entecavir (ETV) treatment in chronic hepatitis $\mathrm{B}(\mathrm{CHB})$ patients with underlying comorbidities such as diabetes mellitus (DM), hypertension, and cirrhosis. Methods: From 2010 to 2012, 116 CHB patients treated with LdT and 578 treated with ETV were compared in this real-practice cohort. The mean changes in eGFR (Modification of Diet in Renal Disease [MDRD] formula) from baseline to months 6,12 , and 18 were analyzed using a linear mixed model. Results: In LdT-treated patients, the mean eGFR increased by $7.6 \%$ at month 18 compared with the eGFR at baseline (MDRD formula in $\mathrm{mL} / \mathrm{min} / 1.73 \mathrm{~m}^{2}$ ). However, in ETV-treated patients, the mean eGFR decreased by $4.1 \%$ at month 18 compared with the eGFR at baseline. In the LdT-treated patients with DM, hypertension, cirrhosis or low eGFR $<90 \mathrm{~mL} / \mathrm{min} / 1.73$ $\mathrm{m}^{2}$, the mean eGFR showed a steady improvement, whereas the mean eGFR was reduced in the same subgroups of ETVtreated patients. Conclusions: The eGFR gradually increased over time during LdT treatment, especially in patients with mild abnormal eGFR at baseline, and in those with DM, hypertension, and cirrhosis, whereas a reduction in eGFR was seen with ETV treatment. (Gut Liver 2015;9:776-783)

Key Words: Telbivudine; Entecavir; Estimated glomerular filtration rate

\section{INTRODUCTION}

Hundreds of million people are chronic hepatitis B virus surface antigen (HBsAg) carriers with serological evidence and 75\% of chronic hepatitis B (CHB) reside in the Asia Pacific region. ${ }^{1}$ Despite the availability of highly effective and safe vaccines for more than 20 years, hepatitis B virus (HBV) infection remains the most common worldwide cause of death from liver disease. ${ }^{2}$

With an expanded range of treatment options and a substantial improvement in the understanding of predictors of response to therapy, the management of patients with $\mathrm{CHB}$ continues to evolve. Currently, there are five oral nucleos(t)ide analogues approved for the treatment of $\mathrm{CHB}$, including three nucleoside analogues (lamivudine, telbivudine, and entecavir), and two nucleotide analogues (adefovir and tenofovir). ${ }^{3}$ Each of these agents showed the effective and profound suppression of viral replication, facilitating hepatitis $\mathrm{B}$ e antigen (HBeAg) seroconversion, achieving alanine aminotransferase (ALT) normalization, and improving liver fibrosis. Telbivudine (LdT) is a specific inhibitor of HBV polymerase which is more potent than adefovir and which was also shown to be superior to lamivudine over 2 years in the GLOBE study, the largest intent-to-treat analysis of long-term HBV nucleoside treatment to date. ${ }^{4,5}$

However, with higher numbers of patients now being treated for $\mathrm{CHB}$, possible adverse events have gained more attention. One area of concern is renal function. CHB is itself important causes of renal disease. In addition to secondary glomerular disease which can be caused by the HBV, treatment of HBV can be potentially nephrotoxic. Adefovir and tenofovir are both acyclic nucleotide analogues structurally, which have been shown to be nephrotoxic. ${ }^{6}$ It has been shown that among a low percentage of human immunodeficiency virus infected patients receiving tenofovir, the estimated glomerular filtration rate (eGFR) decreases, as measured by increases in serum creatine. ${ }^{7}$

In contrast, recent analyses from clinical studies demonstrated

Correspondence to: Sang Hoon Ahn

Department of Internal Medicine, Yonsei University College of Medicine, 50-1 Yonsei-ro, Seodaemun-gu, Seoul 03722, Korea

Tel: +82-2-2228-1936, Fax: +82-2-393-6884, E-mail: AHNSH@yuhs.ac

Received on July 31, 2014. Revised on October 21, 2014. Accepted on November 4, 2014. Published online May 13, 2015

pISSN 1976-2283 eISSN 2005-1212 http://dx.doi.org/10.5009/gnl14297

(.) This is an Open Access article distributed under the terms of the Creative Commons Attribution Non-Commercial License (http://creativecommons.org/licenses/by-nc/4.0) which permits unrestricted non-commercial use, distribution, and reproduction in any medium, provided the original work is properly cited. 
that long-term LdT treatment is associated with steady improvement in renal function, including in-patients with pre-existing renal disease although the potential mechanisms are unclear. ${ }^{8}$ As a view of entecavir (ETV) which is one of recommended antiviral agents, there is no published study indicating this finding and it has been not known whether long-term treatment with ETV affects renal function. Additionally, renal function can be influenced by underlying disease including liver cirrhosis, hypertension, and diabetes mellitus (DM). Therefore, it is important to decide the antiviral agents to protect the renal function for the patients who have the possibility of renal deterioration because of underlying disease. The present study aimed to compare the efficacy and safety of LdT and ETV in patients who received therapy for 18 months according to underlying disease including liver cirrhosis, hypertension, and DM.

\section{MATERIAL AND METHODS}

\section{Patient enrollment}

From 2010 to 2012, 116 CHB patients treated with LdT and 578 with ETV were compared in this real practice cohort. We included patients treated by LdT or ETV more than 18 months and their age was more than 20 years. Exclusion criteria were followings: co-infected with hepatitis $\mathrm{C}$ or human immunodeficiency virus, patient who was pregnant or breastfeeding, patient treated by combination regimen in addition to LdT or ETV, a history of liver transplantation, chronic renal failure patients with hemodialysis or peritoneal dialysis, and patient who was enrolled in another clinical trial. One group was given LdT 600 $\mathrm{mg}$ daily and other group was given ETV $0.5 \mathrm{mg}$ daily.

This study was performed in accordance with the ethical guidelines of the 1975 Declaration of Helsinki. This study was approved by the independent Institutional Review Board of Severance Hospital.

\section{Serum assay}

Analyses of liver function, renal function and creatine kinase level were performed at baseline and at months 6, 12, and 18 of LdT and ETV therapy. HBsAg, HBeAg, antibody to hepatitis B core antigen (anti-HBc), antibody to $\mathrm{HBeAg}$ (anti-HBe) and antibody to HBsAg (anti-HBs) were quantified using radioimmunoassay (Abbott Laboratories, Abbott Park, IL, USA). HBV DNA was measured using the real-time polymerase chain reaction assay on a Cobas TaqMan 48 analyzer (Roche Molecular Systems, Branchburg, NJ, USA) with the lower detection limit of $20 \mathrm{IU} /$ $\mathrm{mL}$. The mean change of eGFR from baseline to 6,12 , and 18 months were analyzed according to the underlying disease including liver cirrhosis, hypertension, and DM. The measurement of eGFR which was based on renal function was assessed using the Modification of Diet in Renal Disease (MDRD) formula for the calculation of eGFR. ${ }^{9}$ We investigated the change of eGFR for the 18 months of follow up. Hypertension was defined ac- cording to by the seventh report of the Joint National Committee and DM was defined as according to the criteria of American Diabetes Association. ${ }^{10,11}$ They were followed up in our hospital for the treatment or clinical observation. Liver cirrhosis was diagnosed using clinical tools including blood test, imaging studies or biopsy. In addition, blood urea nitrogen (BUN) and creatine $(\mathrm{Cr})$ level and other laboratory finding were collected.

\section{Statistical analysis}

Quantitative data were presented as mean \pm standard deviation, categorical data were presented as counts and percentages, and HBV DNA levels were presented as log transformation. The primary endpoint was to estimate the change of eGFR in $\mathrm{CHB}$ patients treated by ETV and LdT, while secondary outcome was to investigate the change of eGFR in patients with DM, hypertension, and liver cirrhosis treated by ETV and LdT. The mean eGFR change compared to baseline eGFR was defined as (eGFR at 18 months-baseline eGFR)/baseline eGFR. Mixed linear Model was used to compare eGFR of patients treated by between ETV and LdT, and chi-square or Fisher exact tests was used for categorical variables. Statistical analyses were performed using SAS version 9.1.3 (SAS, Cary, NC, USA). A two-sided p-value $<0.05$ was considered statistically significant.

\section{RESULTS}

\section{Baseline characteristics}

The mean age was 53 years in ETV group and 54 years in LdT. In the beginning of treatment, the underlying disease including liver cirrhosis (50.9\% vs 51.7\%), hypertension (23.1\% vs $28.4 \%$ ), and DM (18.9\% vs $19.8 \%$ ) were found in ETV and LdT group, respectively. We investigated laboratory result in the beginning of treatment including HBV DNA level, ALT, alanine aspartate aminotransferase (AST), calcium, phosphate, BUN, Cr, eGFR, and total bilirubin. Any result did not show the meaningful difference between two groups. In ETV group, HBeAg positive were 300 (51.6\%) and in LdT group were 55 (44.7\%) ( $p>0.05$ ). The mean ALT value was $85.0 \mathrm{IU} / \mathrm{L}$ and 80.3 in ETV and LdT group, respectively (Table 1). The baseline of mean serum glucose level in patients with DM was $138.1 \pm 72.3 \mathrm{mg} / \mathrm{dL}$ versus $132.1 \pm 77.4 \mathrm{mg} / \mathrm{dL}(\mathrm{p}=0.717)$ and the mean $\mathrm{HbA1c}$ was $7.3 \% \pm 1.7 \%$ versus $7.4 \% \pm 1.4 \%$ in ETV and LdT group ( $\mathrm{p}=0.940$ ), respectively. The mean systolic blood pressure in patients with hypertension was $144.2 \pm 6.3 \mathrm{~mm} \mathrm{Hg}$ versus $144.4 \pm 63.5 \mathrm{~mm} \mathrm{Hg}$ $(\mathrm{p}=0.852)$ and diastolic blood pressure was $85.6 \pm 7.6 \mathrm{~mm} \mathrm{Hg}$ versus $85.0 \pm 8.7 \mathrm{~mm} \mathrm{Hg}(\mathrm{p}=0.655)$ in ETV and LdT group.

\section{Viral response after $\mathbf{1 8}$ months treatment}

For 18 months treatment, HBV DNA level was reduced from $5.6 \log _{10} \mathrm{IU} / \mathrm{mL}$ to $1.5 \log _{10} \mathrm{IU} / \mathrm{mL}$ in ETV group, but from 5.7 $\log _{10} \mathrm{IU} / \mathrm{mL}$ to $2.5 \log _{10} \mathrm{IU} / \mathrm{mL}$ in LdT group. The reduction HBV level compared to baseline HBV DNA level was shown that 
65.1\% was in ETV and 48.4\% in LdT ( $\mathrm{p}=0.004)$.

\section{The mean eGFR change at months 6,12 , and 18 in entire cohort}

In 116 LdT-treated patients, the mean eGFR changes from baseline to months 6,12 , and 18 showed an improvement of 91.4, 91.6, and 94.7 compared to 89.9 of the baseline eGFR level (MDRD formula in $\mathrm{mL} / \mathrm{min} / 1.73 \mathrm{~m}^{2}$ ). However, in 578 ETV-treated patients, the mean eGFR changes from baseline to months 6, 12, and 18 showed an aggravation of 85.5, 85.4, and 84.6 compared to 89.1 of the baseline eGFR level (MDRD formula in $\mathrm{mL} / \mathrm{min} / 1.73 \mathrm{~m}^{2}$ ). They showed the significant different tendency in the change of eGFR level during the antiviral treatment ( $p=0.001$ analyzed by linear mixed model) (Table 2). In

Table 1. Demographic and Other Baseline Characteristics of Patients Treated by Entecavir and Telbivudine

\begin{tabular}{lcc}
\hline \multicolumn{1}{c}{ Characteristic } & ETV $(\mathrm{n}=578)$ & LdT $(\mathrm{n}=116)$ \\
\hline Age, yr & $53.6 \pm 10.9$ & $54.8 \pm 11.3$ \\
Male sex & $402(69.2)$ & $63(54.3)$ \\
Underlying disease & & \\
$\quad$ Liver cirrhosis & $296(51.2)$ & $60(51.7)$ \\
Hypertension & $134(23.1)$ & $33(28.4)$ \\
Diabetes mellitus & $110(18.9)$ & $23(19.8)$ \\
HBV DNA, IU/mL & $2.2 \times 10^{7} \pm 4.4 \times 10^{7}$ & $2.1 \times 10^{7} \pm 4.5 \times 10^{7}$ \\
HBeAg positive/negative & $300(51.6) / 281(48.4)$ & $55(44.7) / 61(52.6)$ \\
ALT, IU/L & $85.0 \pm 65.8$ & $80.3 \pm 78.8$ \\
AST, IU/L & $113.4 \pm 270.3$ & $67.8 \pm 41.4$ \\
Calcium & $8.7 \pm 0.5$ & $8.8 \pm 0.5$ \\
Phosphate & $3.5 \pm 0.6$ & $3.4 \pm 0.5$ \\
Blood urea nitrogen, mg/dL & $14.6 \pm 8.3$ & $13.3 \pm 4.3$ \\
Creatinine, mg/dL & $1.1 \pm 0.7$ & $1.0 \pm 0.3$ \\
eGFR, mL/min/1.73 m ${ }^{2}$ & $89.1 \pm 19.9$ & $89 \pm 18.6$ \\
\hline
\end{tabular}

Continuous values are expressed as mean \pm SD and other values are number (\%).

ETV, entecavir; LdT, telbivudine; HBV, hepatitis B virus; HBeAg, hepatitis B e antigen; ALT, alanine aminotransferase; AST, aspartate aminotransferase; eGFR, estimated glomerular filtration rate (Modification of Diet in Renal Disease formula in $\mathrm{mL} / \mathrm{min} / 1.73 \mathrm{~m}^{2}$ ). the mean eGFR change/baseline eGFR (\%) showed $-4.1 \% \pm 7.6 \%$ in ETV group, but the increase of 7.6 \pm 17.4 was noticed in LdT group (Fig. 1).

\section{The mean eGFR change at months 6,12 , and 18 according to the baseline eGFR}

In subgroup analysis of LdT-treated patients according to the baseline eGFR, the mean eGFR changes from baseline to months 6,12 , and 18 showed different outcomes.

In LdT-treated patients with the low mean baseline eGFR $\left(<90 \mathrm{~mL} / \mathrm{min} / 1.73 \mathrm{~m}^{2}\right)$ showed a significant improvement of $81.0,81.6$, and 86.3 at the time of months 6,12 , and 18 compared to 76.1 of the baseline eGFR level (MDRD formula in $\mathrm{mL}$ / $\min / 1.73 \mathrm{~m}^{2}$ ). However, in LdT-treated patients with the normal mean baseline eGFR showed a slight increase through months 6, 12, and 18. In ETV-treated patients, the mean eGFR changes to months 6,12 , and 18 regardless with low and high baseline showed a eGFR reduction of 75.7, 76.1, 75.1, and 96.8, 96.1,

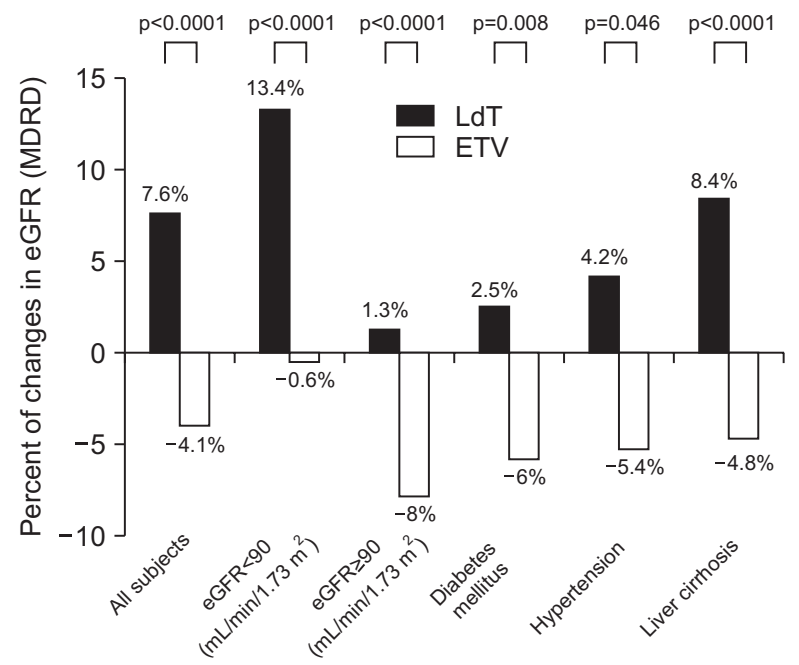

Fig. 1. Analysis for mean change in estimated glomerular filtration rate (eGFR) from baseline to month 18 of therapy by underlying disease. The mean eGFR change compared with baseline eGFR was defined as (eGFR at 18 months-baseline eGFR)/baseline eGFR. MDRD, Modification of Diet in Renal Disease; LdT, telbivudine; ETV, entecavir.

Table 2. The Mean Estimated Glomerular Filtration Rate Change at Months 6, 12, and 18

\begin{tabular}{|c|c|c|c|c|c|c|}
\hline & \multicolumn{2}{|c|}{ Entire cohort } & \multicolumn{2}{|c|}{$\mathrm{eGFR}<90 \mathrm{~mL} / \mathrm{min} / 1.73 \mathrm{~m}^{2}$} & \multicolumn{2}{|c|}{$\mathrm{eGFR} \geq 90 \mathrm{~mL} / \mathrm{min} / 1.73 \mathrm{~m}^{2}$} \\
\hline & $\operatorname{ETV}(n=578)$ & $\operatorname{LdT}(n=116)$ & $\operatorname{ETV}(n=310)$ & $\operatorname{LdT}(\mathrm{n}=61)$ & $\operatorname{ETV}(n=271)$ & $\operatorname{LdT}(n=55)$ \\
\hline Baseline & $89.1 \pm 19.9$ & $89.9 \pm 18.6$ & $75.4 \pm 14.3$ & $76.1 \pm 13.2$ & $104.7 \pm 12.7$ & $103.3 \pm 12.1$ \\
\hline Months 6 & $85.5 \pm 19.7$ & $91.4 \pm 18.6$ & $75.7 \pm 17.4$ & $81.0 \pm 15.7$ & $96.8 \pm 15.8$ & $101.7 \pm 15.9$ \\
\hline Months 12 & $85.4 \pm 20.2$ & $91.6 \pm 20.1$ & $76.1 \pm 18.6$ & $81.6 \pm 16.5$ & $96.1 \pm 16.3$ & $102.8 \pm 17.7$ \\
\hline Months 18 & $84.6 \pm 20.3$ & $94.7 \pm 19.1$ & $75.1 \pm 19.6$ & $86.3 \pm 19.2$ & $95.4 \pm 15.0$ & $104 \pm 14.2$ \\
\hline p-value & \multicolumn{2}{|c|}{$<0.001$} & \multicolumn{2}{|c|}{$<0.001$} & \multicolumn{2}{|c|}{$<0.001$} \\
\hline
\end{tabular}

Values are expressed as mean \pm SD. The change of eGFR between two groups was analyzed by linear mixed model. eGFR, estimated glomerular filtration rate (Modification of Diet in Renal Disease formula in $\mathrm{mL} / \mathrm{min} / 1.73 \mathrm{~m}$ ); ETV, entecavir; LdT, telbivudine. 

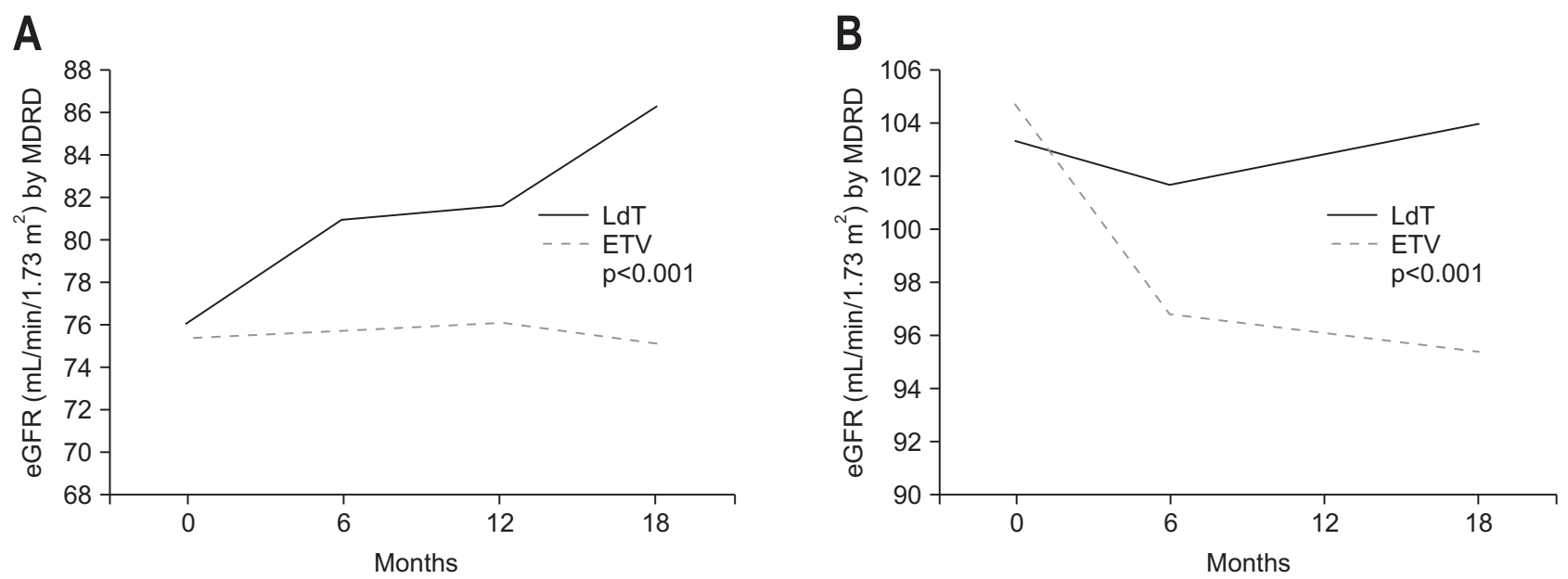

Fig. 2. Evolution of renal function after 18 months of treatment. The black and gray bars indicate estimated glomerular filtration rate (eGFR; mL/ $\min / 1.73 \mathrm{~m}^{2}$ ) with telbivudine (LdT) and entecavir (ETV) treatment, respectively. (A) Patients with baseline eGFR $\leq 90 \mathrm{~mL} / \mathrm{min} / 1.73 \mathrm{~m}{ }^{2} \mathrm{showed} \mathrm{a}$ rise in eGFR with LdT treatment, whereas eGFR declined with ETV therapy. (B) Patients with baseline eGFR $>90 \mathrm{~mL} / \mathrm{min} / 1.73 \mathrm{~m}{ }^{2}$ showed slight increases in eGFR with LdT treatment, whereas eGFR in ETV recipients declined.

MDRD, Modification of Diet in Renal Disease.

95.4, respectively $(\mathrm{p}<0.001$ analyzed by linear mixed model) (Table 2, Fig. 2).

In the subgroup of low eGFR, the mean eGFR change/baseline eGFR (\%) was increased to $13.4 \%$ after 18 months LdT use, but the reduction of $0.6 \%$ was noticed after 18 months ETV use. In the subgroup of high eGFR, the mean eGFR change/baseline eGFR (\%) was reduced to 8.0\% after 18 months ETV use, but the significant change was not found after 18 months LdT use (Fig. 1).

\section{The mean eGFR change at months $6,12,18$ according to the presence of DM}

In subgroup analysis of LdT-treated patients with DM, the mean eGFR changes from baseline to months 6, 12, and 18 showed a different outcomes. In LdT-treated patients with DM showed a significant improvement of 89.0, 88.0, and 90.0 at the time of months 6,12 , and 18 compared to 87.0 of the baseline eGFR level (MDRD formula in $\mathrm{mL} / \mathrm{min} / 1.73 \mathrm{~m}^{2}$ ). In ETVtreated patients, the mean eGFR changes to months 6,12 , and 18 showed a significant aggravation of 79.5, 79.0, and 78.5 in patients with DM compared to 85.5 of the baseline eGFR level (MDRD formula in $\left.\mathrm{mL} / \mathrm{min} / 1.73 \mathrm{~m}^{2}\right)(\mathrm{p}=0.001$ analyzed by linear mixed model) (Table 3, Fig. 3). In the subgroup of DM, the mean eGFR change for 18 months/baseline eGFR (\%) was increased to $2.5 \%$ after 18 months LdT use, but the reduction of 6.0\% was noticed after 18 months ETV use (Fig. 1).

\section{The mean eGFR change at months $6,12,18$ according to the presence of hypertension}

In subgroup analysis of LdT-treated patients with hypertension, the mean eGFR changes from baseline to months 6,12 , and 18 showed a different outcome. In LdT-treated patients with hypertension showed a significant improvement of 86.0, 86.4, and 86.6 at the time of months 6,12 , and 18 compared to 84.2 of the baseline eGFR level (MDRD formula in $\mathrm{mL} / \mathrm{min} / 1.73 \mathrm{~m}^{2}$ ) (Table 3, Fig. 3). In ETV-treated patients, the mean eGFR changes to months 6,12 , and 18 showed a significant aggravation of 74.9, 75.7, and 74.8 in patients with hypertension compare to 80.8 of the baseline eGFR level (MDRD formula in $\mathrm{mL} / \mathrm{min} / 1.73$ $\mathrm{m}^{2}$ ) ( $\mathrm{p}=0.020$ analyzed by linear mixed model) (Table 3$)$. In the subgroup of hypertension, the mean eGFR change/baseline eGFR (\%) was increased to $4.2 \%$ after 18 months LdT use, but the reduction of 5.4\% was noticed after 18 months ETV use (Fig. 1).

\section{The mean eGFR change at months $6,12,18$ according to the presence of liver cirrhosis}

In subgroup analysis of LdT-treated patients with liver cirrhosis, the mean eGFR changes from baseline to months 6,12 , and 18 showed a different outcome. In LdT-treated patients with liver cirrhosis showed a significant improvement of 93.1, 93.6, and 97.5 at the time of months 6, 12, and 18 compared to 91.0 of the baseline eGFR level (MDRD formula in $\mathrm{mL} / \mathrm{min} / 1.73 \mathrm{~m}^{2}$ ) (Table 3, Fig. 3). In ETV-treated patients, the mean eGFR changes to months 6,12 , and 18 showed a significant aggravation of 84.4, 83.8, and 83.4 in patients with liver cirrhosis compared to 88.6 of the baseline eGFR level (MDRD formula in $\mathrm{mL} / \mathrm{min} / 1.73$ $\left.\mathrm{m}^{2}\right)(\mathrm{p}=0.001$ analyzed by linear mixed model) (Table 3, Fig. 3). In the subgroup of liver cirrhosis, the mean eGFR change/baseline eGFR (\%) was increased to $8.4 \%$ after 18 months LdT use, but the reduction of $4.8 \%$ was noticed after 18 months ETV use (Fig. 1).

In liver cirrhosis with Child-Pugh class A, LdT treatment showed the increase of eGFR level of 91.5, 93.1, 93.1, and 97.5 from baseline to months 6,12 , and 18 while ETV treatment showed the decrease of eGFR level of 90.4, 86.1, 86.4, 
Table 3. Subanalysis for the Patients with Diabetes Mellitus, Hypertension, and Liver Cirrhosis: Estimated Glomerular Filtration Rate from Baseline to Months 6, 12, and 18 of Entecavir and Telbivudine

\begin{tabular}{|c|c|c|c|c|c|c|}
\hline & \multicolumn{2}{|c|}{ Diabetes mellitus } & \multicolumn{2}{|c|}{ Hypertension } & \multicolumn{2}{|c|}{ Liver cirrhosis } \\
\hline & ETV $(n=110)$ & $\operatorname{LdT}(\mathrm{n}=23)$ & ETV $(n=134)$ & $\operatorname{LdT}(\mathrm{n}=33)$ & ETV (n=296) & $\operatorname{LdT}(n=60)$ \\
\hline Baseline & $85.5 \pm 26.6$ & $87.0 \pm 25.5$ & $80.8 \pm 25.2$ & $84.2 \pm 22.8$ & $88.6 \pm 20.2$ & $91.0 \pm 14.5$ \\
\hline Months 6 & $79.5 \pm 26.0$ & $89.0 \pm 21.7$ & $74.9 \pm 22.5$ & $86.0 \pm 20.4$ & $84.4 \pm 20.1$ & $93.1 \pm 13.5$ \\
\hline Months 12 & $79.0 \pm 24.5$ & $88.0 \pm 24.9$ & $75.7 \pm 25.1$ & $86.4 \pm 23.0$ & $83.8 \pm 20.4$ & $93.6 \pm 14.9$ \\
\hline Months 18 & $78.5 \pm 25.6$ & $90.0 \pm 26.9$ & $74.8 \pm 25.4$ & $86.6 \pm 23.7$ & $83.4 \pm 21.3$ & $97.5 \pm 16.0$ \\
\hline p-value & \multicolumn{2}{|c|}{0.001} & \multicolumn{2}{|c|}{0.020} & \multicolumn{2}{|c|}{0.001} \\
\hline
\end{tabular}

Values are expressed as mean \pm SD. The change of estimated glomerular filtration rate between two groups was analyzed by linear mixed model. ETV, entecavir; LdT, telbivudine.

A
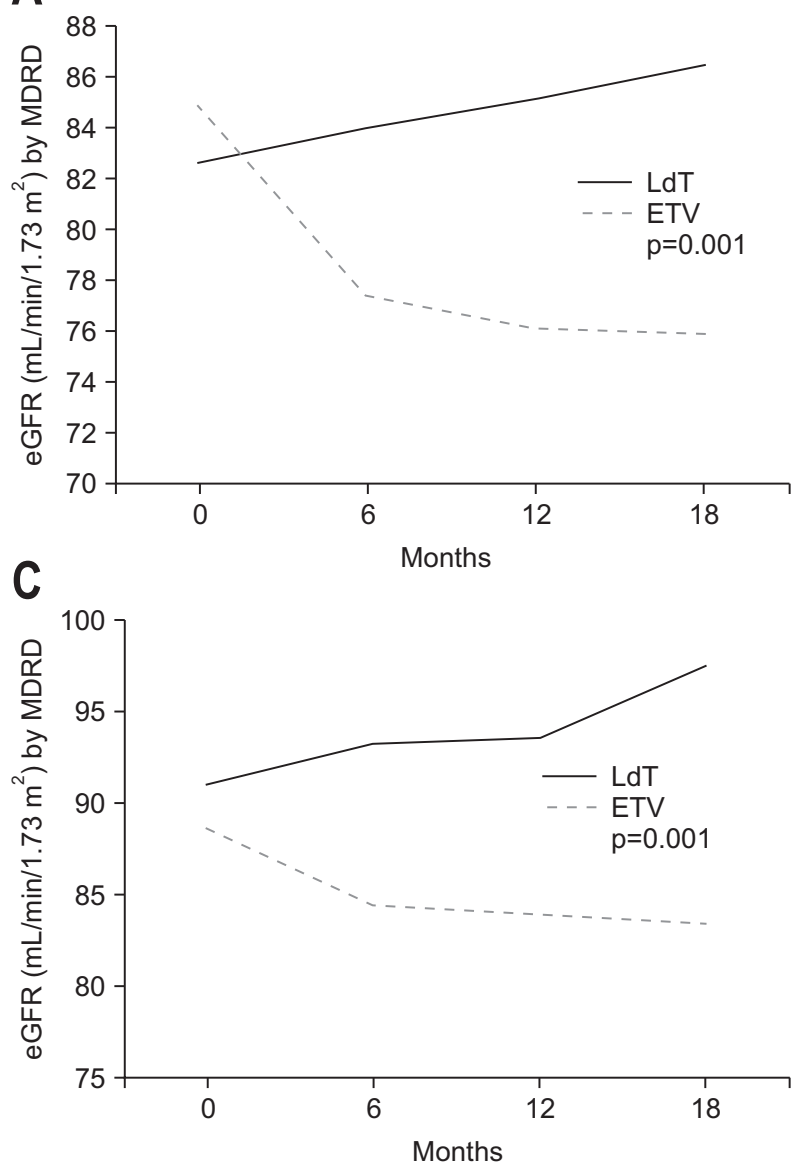

and 85.6 ( $\mathrm{p}<0.001$ analyzed by linear mixed model). In liver cirrhosis with Child-Pugh class B or C, LdT treatment showed the increase of eGFR level of 87.7, 96.6, 93.3, and 97.1 from baseline to months 6,12 , and 18 while ETV treatment showed the decrease of eGFR level of 82.2, 78.4, 74.4, and 75.3 ( $p=0.018$ analyzed by linear mixed model).

\section{DISCUSSION}

Although oral antiviral agents are the first line treatment of CHB and generally well tolerated, some patients have undergone
B

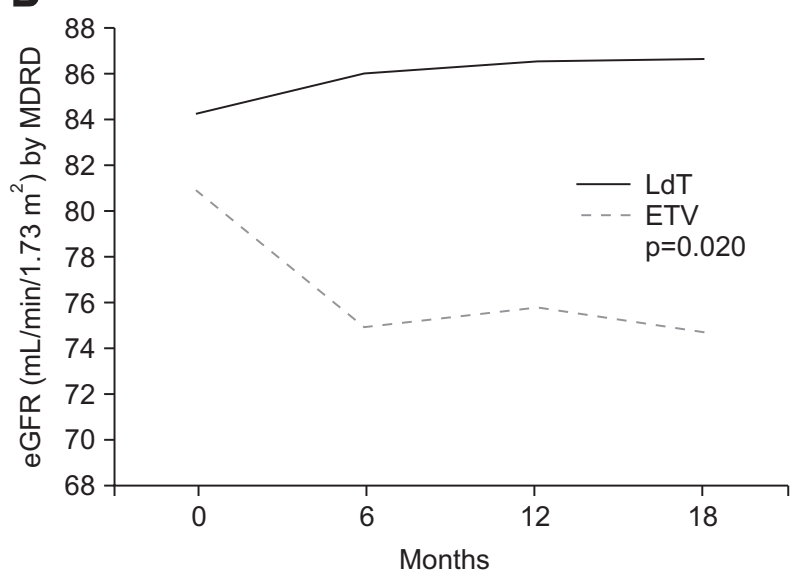

Fig. 3. Evolution of renal function according to underlying disease. The black and gray bars indicate estimated glomerular filtration rate (eGFR; $\mathrm{mL} / \mathrm{min} / 1.73 \mathrm{~m}^{2}$ ) with telbivudine (LdT) and entecavir (ETV) treatment, respectively. The eGFR rose in LdT-treated patients but decreased in patients given ETV. (A) Diabetes mellitus. (B) Hypertension. (C) Cirrhosis. MDRD, Modification of Diet in Renal Disease.

the difficult to use these necessary medication because of adverse events. For example, some antiviral agents can potentially cause adverse events including myopathy, nephropathy, neuropathy, and lactic acidosis. Especially, the effects on muscles including myopathy and creatine kinase elevations have been described with clevudine and LdT use. ${ }^{12,13}$ Adefovir is related with dose-dependent nephropathy, predominantly affecting the proximal renal tubules. ${ }^{14}$ Increased risk of lactic acidosis has also been described for those with impaired liver and renal function taking ETV. ${ }^{15}$ Loss of bone mineral density and hypophosphatemia has been described with the use of nucleotide 
analogues. ${ }^{16}$ However, not all extra-hepatic effects are seriously harmful.

Clinicians take a profound consideration when they adjust the dose of antiviral agents according to the degree of renal impairment because all of the approved antiviral agents undergo renal clearance predominantly in their unchanged forms and some nucleotide analogs are also associated with dose-dependent nephrotoxicity. ${ }^{17}$ Especially at adefovir administration with doses of 10 and $30 \mathrm{mg}$, the efficacy of both groups was similar but renal adverse effects were more common with $30 \mathrm{mg}$. In a study of 80 patients treated with ETV compared to the same number treated with tenofovir, $13.8 \%$ of patients treated with ETV showed serum creatine increase $\geq 0.5 \mathrm{mg} / \mathrm{dL}$ and $8.7 \%$ of patients showed eGFR $<60 \mathrm{~mL} / \mathrm{min}^{18}$

In the REVEAL study, the risk of developing cirrhosis and hepatocellular carcinoma was directly proportional to serum HBV DNA levels. ${ }^{19}$ ETV has been shown to be highly effective at suppressing HBV DNA replication to undetectable levels and normalizing $\mathrm{ALT}^{20,21}$ of note is the very low resistance rate (1.2\%) observed in nucleoside-naive HBeAg-negative patients treated with ETV for up to 5 years. The effective and prolonged suppression of HBV DNA using ETV, which reduces the risk of cirrhosis and HCC, is the primary treatment goal. ${ }^{22,23}$

ETV showed a safety and tolerability in long-term cohort study $^{20,24,25}$ and the fatal renal toxicity has not reported in the real practice. However, the recommendation was suggested for the renal impairment: (1) $\mathrm{Cl}_{\mathrm{cr}} 30$ to $49 \mathrm{~mL} / \mathrm{min}$ : 50\% of usual dose daily or normal dose every 48 hours; (2) $\mathrm{Cl}_{\text {cr }} 10$ to $29 \mathrm{~mL} /$ $\min$ : 30\% of usual dose daily or normal dose every 72 hours; (3) $\mathrm{Cl}_{\mathrm{cr}}<10 \mathrm{~mL} / \mathrm{min}$ (including hemodialysis and peritoneal dialysis): $10 \%$ of usual dose daily or the normal dose every 7 days.

In contrast, recent evidences have suggested a potential renal beneficial effect with the use of LdT. LdT is one of strong potency nucleoside which was established the superiority over lamivudine in $\mathrm{HBeAg}$-positive and $\mathrm{HBeAg}$-negative patients in GLOBE trial although the resistance rate at 2 years for LdT is $11 \%{ }^{26-29}$ Several real-life studies have also shown potential renal improvements that were observed in a broad spectrum of CHB patients. In a randomized double-blinded trial of LdT in decompensated $\mathrm{CHB}$, there was a reduction in serum creatine and increase in eGFR observed after 52 weeks of LdT treatment compared to lamivudine. ${ }^{30}$ This improvement was more marked for those age $>50$ years and for those with reduced GFR (60 to $90 \mathrm{~mL} / \mathrm{min}$ ) at baseline. Of the patients with reduced GFR at baseline (60 to $90 \mathrm{~mL} / \mathrm{min}$ ), $72.3 \%$ moved to GFR >90 $\mathrm{mL} / \mathrm{min}$ after 104 weeks of LdT treatment.

There are limited direct head-to-head trials comparing the different antiviral agents. Although the previous studies compared the efficacy of antiviral drugs, there are many limitations including the small size of patient groups and a heterogeneous baseline which can influence the renal dysfunction. In global trials, long-term LdT was associated with a sustained improve- ment of renal function. ${ }^{8}$ However, it is still unclear in which specific population LdT is associated with the eGFR increase. Hence, we designed this cohort study to assess their efficacy and to verify the change of eGFR according to the underlying disease.

From our analysis, LdT improves eGFR after 18 months of treatment while ETV reduced the eGFR. Further subgroup analysis found that the improvement in renal function becomes apparent including patients with DM, hypertension, and cirrhosis, but the results of a predominant improvement in the first 6 months and steady improvement during the remained 1 year. In the LdT-treated patients with DM, hypertension, cirrhosis or low eGFR of $<90 \mathrm{~mL} / \mathrm{min} / 1.73 \mathrm{~m}^{2}$, the mean eGFR changes from baseline to months 6,12 , and 18 showed a steady improvement.

Tsai et al. ${ }^{31}$ reported LdT was associated with eGFR improvement, especially in patients with renal insufficiency. They insisted there were significantly more patients with eGFR category improvement in the LdT groups at year 1, but there was no significant improvement at year 2. Subgroup analysis of the mean eGFR change showed that both LdT treated with worse baseline eGFR (<90 $\mathrm{mL} / \mathrm{min} / 1.73 \mathrm{~m}^{2}$ ) had better eGFR improvement. Simultaneously, in treated by LdT of $271 \mathrm{CHB}$ patients and by ETV of $310 \mathrm{CHB}$ patients with a $\leq 90 \mathrm{~mL} / \mathrm{min} / 1.73 \mathrm{~m}^{2}$ of eGFR, the change in eGFR was $13.4 \%$ and $-0.6 \%$, respectively. In our comparison between LdT $(n=55)$ and ETV $(n=61)$ with a $>90$ $\mathrm{mL} / \mathrm{min} / 1.73 \mathrm{~m}^{2}$ of eGFR, a higher decline from baseline was noted in the ETV group while in the LdT group increased with mild improvement (-8.0\% vs 1.3\%, respectively). Therefore, LdT is thought not to increase additionally the eGFR in patients with $>90 \mathrm{~mL} / \mathrm{min} / 1.73 \mathrm{~m}^{2}$ of eGFR.

Special attention should be given to patients with a history of pre-existing renal insufficiency or other renal disease risks from comorbidities, because they are at increased risk of developing changes in renal function during prolonged CHB therapy. The major clinical relevance of accurate eGFR monitoring in DM patients has been further confirmed by recent evidence that low GFR was a strong risk factor for the prognosis related with vascular disease and the regular checkup of eGFR was recommended in type $2 \mathrm{DM}^{32,33}$ In our study, LdT also showed significant improvement of eGFR in the group with DM. In DM with CHB patients, GFR was increased in LdT compared to ETV. Those taking LdT ( $\mathrm{n}=23$ ) and ETV ( $\mathrm{n}=110)$ had a $2.5 \%$ and $-6.0 \%$ GFR change, respectively.

Hypertension is commonly present in patients with chronic kidney disease and the prevalence of hypertension varied inversely with GFR. ${ }^{34,35}$ In our study, LdT also showed significant improvement of eGFR in patients with hypertension. The $4.2 \%$ increase of eGFR compared to the baseline eGFR was found at months 18. However, in patients treated by ETV in the group with hypertension, 5.4\% of eGFR was reduced compared to the baseline eGFR.

Renal dysfunction is a common complication of cirrhosis and 
confers a poor prognosis. ${ }^{36}$ Renal dysfunction occurs in 20\% of patients with cirrhosis admitted to hospital, often linked with other complications of cirrhosis such as variceal bleeding and spontaneous bacterial peritonitis. ${ }^{37}$ In a systematic review of 118 studies, the presence of renal dysfunction was a powerful predictor of death in decompensated cirrhosis. ${ }^{38}$ In our study, LdT also showed significant improvement of eGFR in the group with liver cirrhosis. The $8.4 \%$ of increase compared to the baseline eGFR was found. However, in patients treated by ETV in the group with liver cirrhosis, 4.8\% of eGFR was reduced compared to the baseline eGFR. In further stratified by Child-Pugh class, eGFR in patients with Child-Pugh class B or C as well as ChildPugh class A showed the improvement. In the point that renal function is one of important prognostic factors for decompensated liver cirrhosis, this phenomenon was a noticeable finding to make a plan for the treatment of $\mathrm{CHB}$ with liver cirrhosis.

Serum creatine is one of three variables comprising the model for end-stage liver disease score which is widely used in predicting short term mortality in allocating priority for orthotopic liver transplantation. ${ }^{39}$ Although eGFR was changed through 18 months, $\mathrm{Cr}$ has not been changed significantly compared to the baseline. It might be associated with the baseline $\mathrm{Cr}$ in patients were not abnormally low. It should be validated in more extended population with abnormal $\mathrm{Cr}$ and eGFR.

We have some limitation in this report. First, it is a retrospective study. Second, we described the result that LdT increased the eGFR without any pathophysiologic theory. Although LdT treatment can affect angiotensin converting enzyme that can control renin-angiotensin aldosterone regulatory system in previous report, ${ }^{40}$ further investigations were required for this phenomenon under the pharmaco-pathophysiology.

In conclusion, GFR measurements may help in identifying subjects at risk of progression even before the onset of nephropathy. The eGFR has been gradually increased over time during LdT treatment especially in patients with abnormal eGFR at baseline, DM, hypertension, and liver cirrhosis while the reduction of eGFR was seen with ETV treatment. The safety of nucleos(t)ide should be considered importantly because it will require long-term treatment and influence renal function. The further studies were required for the investigation of pathophysiology of LdT therapy to increase eGFR.

\section{CONFLICTS OF INTEREST}

No potential conflict of interest relevant to this article was reported.

\section{ACKNOWLEDGEMENTS}

This study was supported by the Liver Cirrhosis Clinical Research Center, in part by a grant from the Korea Healthcare Technology R\&D project, Ministry of Health and Welfare, Re- public of Korea (number: HI10C2020). The funders had no role in study design, data collection and analysis, decision to publish, or preparation of the manuscript.

\section{REFERENCES}

1. Liaw YF, Chu CM. Hepatitis B virus infection. Lancet 2009;373: 582-592.

2. Perz JF, Armstrong GL, Farrington LA, Hutin YJ, Bell BP. The contributions of hepatitis B virus and hepatitis $C$ virus infections to cirrhosis and primary liver cancer worldwide. J Hepatol 2006; 45:529-538.

3. Yuen MF, Lai CL. Treatment of chronic hepatitis B: evolution over two decades. J Gastroenterol Hepatol 2011;26 Suppl 1:138-143.

4. Chan HL, Heathcote EJ, Marcellin P, et al. Treatment of hepatitis B e antigen positive chronic hepatitis with telbivudine or adefovir: a randomized trial. Ann Intern Med 2007;147:745-754.

5. Liaw YF, Gane E, Leung N, et al. 2-Year GLOBE trial results: telbivudine is superior to lamivudine in patients with chronic hepatitis B. Gastroenterology 2009;136:486-495.

6. Hadziyannis SJ, Tassopoulos NC, Heathcote EJ, et al. Long-term therapy with adefovir dipivoxil for HBeAg-negative chronic hepatitis B for up to 5 years. Gastroenterology 2006;131:1743-1751.

7. Mauss S, Berger F, Schmutz G. Antiretroviral therapy with tenofovir is associated with mild renal dysfunction. AIDS 2005;19:9395.

8. Gane EJ, Deray G, Liaw YF, et al. Telbivudine improves renal function in patients with chronic hepatitis B. Gastroenterology 2014;146:138-146.

9. Levey AS, Bosch JP, Lewis JB, Greene T, Rogers N, Roth D. A more accurate method to estimate glomerular filtration rate from serum creatinine: a new prediction equation. Modification of Diet in Renal Disease Study Group. Ann Intern Med 1999;130:461470.

10. Cuddy ML. Treatment of hypertension: guidelines from JNC 7 (the seventh report of the Joint National Committee on Prevention, Detection, Evaluation, and Treatment of High Blood Pressure 1). J Pract Nurs 2005;55:17-21.

11. American Diabetes Association. Standards of medical care in diabetes: 2011. Diabetes Care 2011;34 Suppl 1:S11-S61.

12. Zou XJ, Jiang XQ, Tian DY. Clinical features and risk factors of creatine kinase elevations and myopathy associated with telbivudine. J Viral Hepat 2011;18:892-896.

13. Kim BK, Oh J, Kwon SY, et al. Clevudine myopathy in patients with chronic hepatitis B. J Hepatol 2009;51:829-834.

14. Ha NB, Ha NB, Garcia RT, et al. Renal dysfunction in chronic hepatitis B patients treated with adefovir dipivoxil. Hepatology 2009;50:727-734.

15. Lange CM, Bojunga J, Hofmann WP, et al. Severe lactic acidosis during treatment of chronic hepatitis B with entecavir in patients with impaired liver function. Hepatology 2009;50:2001-2006.

16. Schiefke I, Fach A, Wiedmann M, et al. Reduced bone mineral 
density and altered bone turnover markers in patients with noncirrhotic chronic hepatitis B or C infection. World J Gastroenterol 2005;11:1843-1847.

17. Izzedine H, Hulot JS, Launay-Vacher V, et al. Renal safety of adefovir dipivoxil in patients with chronic hepatitis B: two doubleblind, randomized, placebo-controlled studies. Kidney Int 2004;66: 1153-1158.

18. Gish RG, Clark MD, Kane SD, Shaw RE, Mangahas MF, Baqai S. Similar risk of renal events among patients treated with tenofovir or entecavir for chronic hepatitis B. Clin Gastroenterol Hepatol 2012;10:941-946.

19. Iloeje UH, Yang HI, Su J, et al. Predicting cirrhosis risk based on the level of circulating hepatitis B viral load. Gastroenterology 2006;130:678-686.

20. Chang TT, Gish RG, de Man R, et al. A comparison of entecavir and lamivudine for HBeAg-positive chronic hepatitis B. N Engl J Med 2006;354:1001-1010.

21. Kwon DH, Kim IH, Choung BS, et al. Continuous long-term entecavir therapy in naive chronic hepatitis B patients showing partial virologic response. Gut Liver 2013;7:712-718.

22. Kim MN, Kim SU, Kim BK, et al. Long-term changes of liver stiffness values assessed using transient elastography in patients with chronic hepatitis B receiving entecavir. Liver Int 2014;34:12161223.

23. Kim MN, Kim SU, Park JY, et al. Risk assessment of liver-related events using transient elastography in patients with chronic hepatitis B receiving entecavir. J Clin Gastroenterol 2014;48:272-278.

24. Chang TT, Lai CL, Kew Yoon S, et al. Entecavir treatment for up to 5 years in patients with hepatitis B e antigen-positive chronic hepatitis B. Hepatology 2010;51:422-430.

25. Gish RG, Lok AS, Chang TT, et al. Entecavir therapy for up to 96 weeks in patients with HBeAg-positive chronic hepatitis B. Gastroenterology 2007;133:1437-1444.

26. Lai CL, Gane E, Liaw YF, et al. Telbivudine versus lamivudine in patients with chronic hepatitis B. N Engl J Med 2007;357:25762588.

27. Zeuzem S, Gane E, Liaw YF, et al. Baseline characteristics and early on-treatment response predict the outcomes of 2 years of telbivudine treatment of chronic hepatitis B. J Hepatol 2009;51:11-20.

28. Park H, Park JY, Kim SU, et al. Efficacy of switching to telbivudine plus adefovir in suboptimal responders to lamivudine plus adefovir. World J Gastroenterol 2013;19:7671-7679.

29. Ahn SH, Kweon YO, Paik SW, et al. Telbivudine in combination with adefovir versus adefovir monotherapy in HBeAg-positive, lamivudine-resistant chronic hepatitis B. Hepatol Int 2012;6:696706.

30. Chan HL, Chen YC, Gane EJ, et al. Randomized clinical trial: efficacy and safety of telbivudine and lamivudine in treatment-naive patients with HBV-related decompensated cirrhosis. J Viral Hepat 2012;19:732-743.

31. Tsai MC, Lee CM, Chiu KW, et al. A comparison of telbivudine and entecavir for chronic hepatitis B in real-world clinical practice. J Antimicrob Chemother 2012;67:696-699.

32. Nishimura M, Kato Y, Tanaka T, et al. Significance of estimating the glomerular filtration rate for the management of hypertension in type 2 diabetes with microalbuminuria. Hypertens Res 2013;36:705-710.

33. American Diabetes Association. Standards of medical care in diabetes: 2014. Diabetes Care 2014;37 Suppl 1:S14-S80.

34. Whaley-Connell AT, Sowers JR, Stevens LA, et al. CKD in the United States: Kidney Early Evaluation Program (KEEP) and National Health and Nutrition Examination Survey (NHANES) 19992004. Am J Kidney Dis 2008;51:S13-S20.

35. Buckalew VM Jr, Berg RL, Wang SR, Porush JG, Rauch S, Schulman G. Prevalence of hypertension in 1,795 subjects with chronic renal disease: the modification of diet in renal disease study baseline cohort. Modification of Diet in Renal Disease Study Group. Am J Kidney Dis 1996;28:811-821.

36. du Cheyron D, Bouchet B, Parienti JJ, Ramakers M, Charbonneau P. The attributable mortality of acute renal failure in critically ill patients with liver cirrhosis. Intensive Care Med 2005;31:1693-1699.

37. Garcia-Tsao G, Parikh CR, Viola A. Acute kidney injury in cirrhosis. Hepatology 2008;48:2064-2077.

38. D’Amico G, Garcia-Tsao G, Pagliaro L. Natural history and prognostic indicators of survival in cirrhosis: a systematic review of 118 studies. J Hepatol 2006;44:217-231.

39. Al Sibae MR, Cappell MS. Accuracy of MELD scores in predicting mortality in decompensated cirrhosis from variceal bleeding, hepatorenal syndrome, alcoholic hepatitis, or acute liver failure as well as mortality after non-transplant surgery or TIPS. Dig Dis Sci 2011;56:977-987.

40. Liang KH, Chen YC, Hsu CW, Chang ML, Yeh CT. Decrease of serum angiotensin converting enzyme levels upon telbivudine treatment for chronic hepatitis B virus infection and negative correlations between the enzyme levels and estimated glumerular filtration rates. Hepat Mon 2014;14:e15074. 